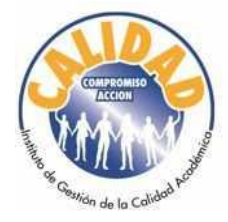

Vicerrectoría Académica

Instituto de Gestión de la Calidad Académica

Co-creando Excelencia

http://revistas.uned.ac.cr./index.php/revistacalidad

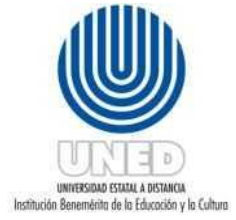

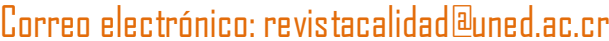

\title{
Estrategias docentes para la promoción del aprendizaje autónomo en estudiantes universitarios que utilizan plataformas LMS
}

\section{Teaching strategies to promote autonomous learning in university students by means of Learning Management Systems (LMS)}

\author{
$\frac{\text { DOl: http://dx.doi.org/10.22458/caes.v10i2.2715 }}{\text { Volumen 10, Número } 2}$ \\ 30 de noviembre de 2019 \\ pp. $215-246$
}

Ana Lorena Vargas-Cubero ${ }^{1}$ .avargas@uned.ac.cr

Universidad Estatal a Distancia, Costa Rica Gabriela Villalobos-Torres ${ }^{2}$ gvillalobost@uned.ac.cr Universidad Estatal a Distancia, Costa Rica

Recibido: 29 de enero de 2019

Aprobado: 10 de setiembre de 2019

${ }^{1}$ Especialista en evaluación educativa del Posgrado de la Universidad de Costa Rica. Graduada del Doctorado en Tecnología Instruccional y Educación a Distancia de la NOVA Sowteastern University de la Florida. Actualmente, se desempeña como coordinadora de la Cátedra de Ambiente, Política y Sociedad de la Universidad Estatal a Distancia, además es profesora de la Universidad de Costa Rica. Ha realizado investigaciones y publicaciones en temáticas propias de las Educación a Distancia y la tecnología como medio de aprendizaje, entre otras. Universidad Estatal a Distancia, Costa Rica. avargas@uned.ac.cr, http://orcid.org/0000-0001-5513-6851

2 Especialista en prevención y atención de la violencia intrafamiliar y de género del Posgrado de la Universidad de Costa Rica y egresada del posgrado en Psicología Laboral de la Universidad Autónoma de Centroamérica. Actualmente se desempeña como coordinadora de la Cátedra de Trabajo Social de la Universidad Estatal a Distancia; así mismo es coordinadora del Observatorio Latinoamericano de Trata y Tráfico de Personas Capítulo Costa Rica. Ha realizado investigaciones y publicaciones en temas asociados a la educación a distancia y tecnología para el aprendizaje, violencias, trata de personas y tráfico ilícito de migrantes, entre otros. Universidad Estatal a Distancia, Costa Rica, gvillalobost@uned.ac.cr, http://orcid.org/0000-0002-0650-8978

Estrategias docentes para la promoción del aprendizaje autónomo en estudiantes universitarios que utilizan plataformas LMS

Ana Lorena Vargas-Cubero, Gabriela Villalobos-Torres

DOI: http://dx.doi.org/10.22458/caes.v10i2.2715

\section{(c) (i) (8)}




\section{Resumen}

El aprendizaje autónomo en estudiantes universitarios que utilizan plataformas LMS implica el conocimiento teórico del concepto, así como la puesta en práctica de diversas estrategias que fomenten el aprendizaje autónomo en estos entornos. Es así como en un estudio realizado con docentes de asignaturas de la Escuela de Ciencias Sociales y Humanidades y estudiantes que actualmente cursan asignaturas a distancia híbridas, es decir que utilizan recursos y realizan algunas actividades en LMS se planteó el siguiente problema ¿de qué manera las y los docentes impulsan estrategias que promueven el aprendizaje autónomo en estudiantes de un modelo educativo a distancia que utilizan Learning Management System (LMS)? Los resultados obtenidos versan en un conocimiento por parte de docentes acerca del concepto de aprendizaje autónomo, lo cual concuerda con los postulados teóricos al respecto. Así mismo, se detallan características que identifican a un estudiante que aprende con autonomía y las estrategias empleadas por docentes para promover el aprendizaje autónomo mediante las plataformas LMS, mismas que contribuyen a obtener tanto resultados positivos como negativos y diversas manifestaciones en estudiantes sobre la aplicación de las mismas. Las conclusiones discurren sobre la importancia del trabajo colaborativo para el aprendizaje autónomo, la falta de conocimiento metodológico y aplicación de las estrategias en asignaturas a través de plataformas LMS y la necesidad de procesos de capacitación para minimizar el sesgo existente.

Palabras clave: Educación a distancia - LMS - aprender en autonomía - aprender a aprender - aprendizaje significativo

\section{Abstract}

Autonomous learning in university students using LMS platforms implies theoretical knowledge of the concept, as well as the implementation of various strategies that promote autonomous learning in these environments. Thus is how in a study conducted with subject teachers from the School of Social Sciences and Humanities and students currently studying hybrid distance subjects, that is, they use resources and perform some activities in LMS, it was raised as a problem of how and teachers promote strategies that promote autonomous learning in students of a distance education model that use Learning Management System (LMS)?The results obtained are related to the teachers knowledge about the concept of autonomous learning, which is consistent with the theoretical postulates in this regard. Likewise, the characteristics that identify a student who learns with autonomy and the strategies used by teachers to promote autonomous learning through LMS platforms, which contribute to both positive and negative results, and various manifestations in students are

Estrategias docentes para la promoción del aprendizaje autónomo en estudiantes universitarios que utilizan plataformas LMS

Ana Lorena Vargas-Cubero, Gabriela Villalobos-Torres DOI: http://dx.doi.org/10.22458/caes.v10i2.2715

\section{(c) (P)}


detailed on the application thereof. The conclusions discuss the importance of collaborative work for autonomous learning, the lack of methodological knowledge and the application of course strategies through LMS platforms and the need for training processes to minimize existing bias

Keywords: Distance education - LMS - learning autonomy - learning to learn - meaningful learning

\section{Introducción}

La Universidad en que se realizó la investigación posee un sistema educativo a distancia, el cual se caracteriza porque quien aprende y quienes enseñan están separados físicamente, no obstante, el estudiantado es apoyado por una institución mediante la cual se le ofrecen recursos, materiales, instrucciones, comunicación e interacción por distintos medios, todo ello con el fin de facilitarle experiencias de aprendizaje con significatividad (Simonson, Smaldino, Albright \& Zvacek, 2006).

La institución señala desde su modelo pedagógico (UNED, Consejo Universitario, 2004) que aprender a aprender es el objetivo más ambicioso e irrenunciable de la educación a distancia. Lo que se plantea entonces es que quien estudia debe ser capaz de construir aprendizajes con significatividad, es decir aquellos a los que pueda atribuirles sentido personal de modo tal que progresivamente sea el estudiantado quien tome el control de su proceso de adquisición de conocimientos (Coll, Mauri, \& Onrubia, 2006), para ello es innegable la importancia de las estrategias cognitivas utilizadas, las cuales se definen como los procedimientos que utiliza el estudiantado para el logro de la meta de aprendizaje, es entonces un plan de acción que implica como resultado un aprendizaje (Esteban \& Zapata, 2016).

Estrategias docentes para la promoción del aprendizaje autónomo en estudiantes universitarios que utilizan plataformas LMS

Ana Lorena Vargas-Cubero, Gabriela Villalobos-Torres

DOI: http://dx.doi.org/10.22458/caes.v10i2.2715

\section{(c) (i) $(5)$}


Es así como las estrategias cognitivas se conciben como el trabajo intelectual que realiza quien estudia y que le permite adquirir, codificar y recuperar la información. Por ende intervienen en la forma como se aprende en términos de la gama de situaciones y circunstancias en que estas se desarrollan, así como la forma en que el estudiantado planifica y regula sus actividades (Universidad Estatal a Distancia, Consejo Universitario, 2004), lo que conduce a pensar en un aprendiz que conlleva su proceso de aprendizaje de manera autónoma e independiente; es decir, quien estudia asume la responsabilidad para motivarse a aprender y a comprender que es un proceso del individuo, en tanto que quien aprende puede planear y realizar actividades autocontroladas, así como evaluarlas críticamente en cuanto al logro de aprendizajes obtenidos, es el fin involucrarse de manera activa en su proceso de aprendizaje, supervisar y controlar su progreso (Varela-Ruiz, 2009).

Aunado a lo anterior, en la UNED en el año 2004 desde su modelo pedagógico se define el aprendizaje autónomo como la forma que tiene una persona para dirigir, controlar, regular y valorar su forma de aprender, ello de modo consciente y con intención deliberada de seguir el proceso mediante la utilización de estrategias de aprendizaje para alcanzar el objetivo o meta deseada. Esto es propio de alguien que tiene control metacognitivo, tal y como se define en Osses y Jaramillo (2008), quienes plantean que este responde al "cómo", tiende a la autorregulación, quien aprende participa activamente de su proceso, es capaz de dirigir su camino hacia el aprendizaje y no solo esperar o reaccionar a estímulos externos, pues al aprender controla y gestiona su aprendizaje (Arguelles y Nagles 2007).

Se señala, por parte de esta Universidad, donde se practica un modelo de educación a distancia, que para el logro del control cognitivo y de aprendizaje en autonomía se requieren herramientas y estrategias que la misma institución debe proveer (UNED, Consejo Universitario, 2004). Las herramientas y estrategias serían las formas, medios, acciones y

Estrategias docentes para la promoción del aprendizaje autónomo en estudiantes universitarios que utilizan plataformas LMS

Ana Lorena Vargas-Cubero, Gabriela Villalobos-Torres DOI: http://dx.doi.org/10.22458/caes.v10i2.2715

\section{(c) (i) (8)}


procedimientos de que dispone quien aprende para conocer sus procesos mentales y saber cómo utilizarlos o adaptarlos para el logro de las metas de aprendizaje propuestas (Osses y Jaramillo, 2008). Así el estudiantado participa activamente, elabora representaciones mentales y desarrolla procesos internos derivados de su relación con el entorno, así dilucida y reinterpreta la realidad de manera continua y dinámica, a partir de sus estructuras cognitivas (UNED, Consejo Universitario, 2004).

Por las razones anteriores, el Consejo Universitario (2004) establece que el aprendizaje en autonomía debe promoverse desde la interrelación estudiante-docente, de modo que el profesorado posee el rol de orientador del aprendizaje autónomo y de aprender a aprender, supone un aprendiz que es capaz de gestionar su proceso de aprendizaje, de utilizar las herramientas y estrategias que tiene a disposición para producir conocimiento y lograr la meta educativa propuesta. Competencias del Siglo XXI define aprender a aprender como una capacidad presente en las personas que perciben sus mecanismos de aprendizaje, controlan su desempeño para reorientarlo, mejorarlo y corregirlo si es necesario (2014). La adquisición de nuevos conocimientos se traslada más allá del ámbito académico ya que trasciende hasta el contexto personal y laboral; aspectos que deben facilitarse en todas las modalidades de asignaturas que ofrece la UNED (UNED, 2004).

En la institución en que se realizó el estudio se ha incursionado desde hace algunos años en el uso de LMS (Learning Management System) para impartir asignaturas de modo híbrido (con componentes en línea y a distancia tradicional) o totalmente virtual (asignaturas que se desarrollan completamente en entornos virtuales), no obstante, al contar con las nuevas herramientas se adquiere el reto de saber cómo utilizarlas de manera que realmente mejoren la enseñanza y el aprendizaje (Fernández, 2009).

Estrategias docentes para la promoción del aprendizaje autónomo en estudiantes universitarios que utilizan plataformas LMS

Ana Lorena Vargas-Cubero, Gabriela Villalobos-Torres DOI: http://dx.doi.org/10.22458/caes.v10i2.2715

\section{(c) (i) $\$$}


Por tanto, para un logro real de aprendizajes se requiere del apoyo docente para una adecuada mediación que genere aprendizajes con significatividad, donde el estudiantado tenga un rol protagónico en el que no solo adquiera contenidos temáticos, sino que logre ser autónomo y que consiga "aprender a aprender"; es decir, que no sea un sujeto pasivo, sino que gestiones su aprendizaje y sea autorregulado en tanto tenga control sobre su proceso de adquisición de conocimientos con una orientación clara hacia la tarea, motivado al logro y con la capacidad de no estancarse, sino de superar por sí mismo los obstáculos que se le puedan presentar y buscar los apoyos necesarios en distintas y diversas fuentes, un estudiante que sea capaz de mejorar a partir de los errores (Vargas 2017).

Por lo anterior, se busca investigar de qué manera las personas docentes desarrollan estrategias para que quienes estudian mediante plataformas LMS, en esta Universidad, desarrollen el potencial para aprender a aprender de modo autónomo y significativo (UNED, Vicerrectoría Académica, 2004). Lo anterior en virtud de que es fundamental examinar la forma en que el docente asume prácticas de estrategias encaminadas a lograr el aprendizaje autónomo en el estudiantado, lo que le llevará a superar las barreras que pueden resultar del estudio a distancia, tales como adaptación al nuevo contexto, el desarrollo del conocimiento y una manera de aprender diferente a la acostumbrada, así como aprender a relacionarse de manera distinta a la tradicional con compañeros y profesores.

\section{Referentes teóricos}

El estudiante que aprende en autonomía se caracteriza por contarse entre aquellos que se "evalúan y se dirigen a sí mismos" (Moore, 1972, p. 87). Estas personas deben asumir cierto grado de dirección y control de sí mismo en el proceso de aprender. Quien aprende así logrará ser autorregulado, este tipo de estudiante se define como aquel que es capaz de utilizar estrategias que le lleven al logro de los objetivos planteados, siempre buscando los

Estrategias docentes para la promoción del aprendizaje autónomo en estudiantes universitarios que utilizan plataformas LMS

Ana Lorena Vargas-Cubero, Gabriela Villalobos-Torres DOI: http://dx.doi.org/10.22458/caes.v10i2.2715

\section{(c) (i) (8)}


apoyos necesarios para ello y no quedarse en el obstáculo, sino que pueda planificar su proceso del logro en pleno conocimiento de la tarea y con el objetivo de realizarla de manera exitosa, lo que implica que poseen un alto compromiso hacia las metas académicas y una percepción de autoeficacia que le llevan a tomar acciones para el logro de las tareas determinadas (Zimmerman y Martínez-Pons, 1986).

Es así como esta autonomía en el aprendizaje y la autorregulación deben entonces ser el fin último de la educación (Manrique, 2004) y se expresa en aprender a aprender, con lo que quien aprende es quien promueve las acciones para conocer sus necesidades instruccionales, establecer sus metas de aprendizaje y las respectivas estrategias para el logro de las mismas, así como las formas de evaluarlas (Writt \& Perkins, 2008).

Lo anterior se relaciona directamente con el cognitivismo, el cual plantea que el ser humano debe ser considerado no como un depositario de información, sino como un procesador de esta (Bruner, 1983). Se puede plantear que existe evidencia de que este aspecto es determinante para el aprendizaje, pues quienes estudian son entes activos que selecciona la información y elaboran nuevos significados a partir de conocimientos construidos socialmente tornándose en gestores del conocimiento, para lo cual se requiere de habilidades cognitivas que apuntan a las distintas capacidades intelectuales para realizar las tareas propuestas, las cuales pueden ser variadas y numerosas y afectarse por la tarea misma, la actitud del aprendiz o por otras variables del entorno. Ejemplos de las habilidades cognitivas son la memoria, la atención, la concentración, la lectura, entre otras. (Betini, Contini \& Castro, 2010). Por su parte Hartman y Sternberg (1993) han Ilamado a estas habilidades los obreros (workers) del conocimiento.

Estrategias docentes para la promoción del aprendizaje autónomo en estudiantes universitarios que utilizan plataformas LMS

Ana Lorena Vargas-Cubero, Gabriela Villalobos-Torres DOI: http://dx.doi.org/10.22458/caes.v10i2.2715

\section{(c) (i) $(5)$}


Por otra parte, en educación a distancia se plantea que este modelo educativo supone la separación profesor-estudiante y el uso de tecnologías colaborativas que mediadas adecuadamente pueden potenciar la promoción del aprendizaje autónomo (García, 2006). Tal y como lo señalan Salas y Umaña (2011) en concordancia con Clark (2001), el uso de medios tecnológicos por sí mismos no son generadores de aprendizaje, sino que son solo vehículos de información y mecanismos de entrega de contenidos, pero por sí mismos no influyen en el aprendizaje, sino que hay otros aspectos por considerar, entre ellos mencionan la autoras al diseño del entorno de aprendizaje, la consideración de los conocimientos previos del estudiantado y la mediación pedagógica. "En otras palabras, aplicando esto a los medios en general, de nada sirve el medio y todo su potencial (sistema simbólico, sistema de procesamiento, capacidad expresiva y pertinencia pedagógica) si no se es capaz de implementar un buen método y un ambiente de aprendizaje significativo" (Salas y Umaña, 2011, p. 21)

Así mismo, se presume que debe darse un proceso mediado por tecnologías y una organización institucional que respalde el logro de las metas educativas del estudiantado (Lasser, 2018) y además la presencia de un rol docente que promueven el aprendizaje autónomo y autorregulado, de modo tal que quien aprenda sea capaz de hacerlo de manera estratégica y con significatividad (Valenzuela, 2000) y que, como ya se ha mencionado a lo largo de este escrito, la autonomía se traduzca en la capacidad de hacer uso de las herramientas y fuentes adecuadas para gerenciar su aprendizaje sin depender siempre de la persona docente que dirija su proceso, así como que la autorregulación se evidencie en un estudiantado con control metacognitivo, enfocado en la tarea, motivado, aprendiente de sus errores, enfocado en la mejora y en el logro de aprendizajes con significatividad (Vargas, 2017).

Estrategias docentes para la promoción del aprendizaje autónomo en estudiantes universitarios que utilizan plataformas LMS

Ana Lorena Vargas-Cubero, Gabriela Villalobos-Torres DOI: http://dx.doi.org/10.22458/caes.v10i2.2715

\section{(c) (1) \$}


Al respecto, el docente no desaparece, sino que se asume como un orientador en un contexto en donde el estudiante a distancia en muchas ocasiones se siente desmotivado debido a la falta de apoyo docente y de retroalimentación por parte de este, además se autoconcibe aislado de su institución educativa, de profesores, compañeras y compañeros, siente incertidumbres acerca de su aprendizaje y su progreso. Por ende, el apoyo a los estudiantes a distancia no debe ser tomado como algo de poca importancia, pues pueden desarrollar sentimientos de alienación, aislamiento, exclusión, insuficiencia, falta de confianza y de seguridad en sus capacidades (Gallusa, 1998). Lo anterior no es menos cierto para quienes estudian a distancia mediante plataformas de aprendizaje LMS, por cuanto esos mismos han manifestado que el uso de plataformas educativas en línea puede favorecer el aprendizaje, "siempre que el recurso esté apoyado por una adecuada interacción y mediación por parte de quien ejerce la docencia" (Vargas y Villalobos, 2018, p. 17).

En este sentido, la interacción hace alusión a las relaciones docente - estudiante y viceversa y entre estudiantes, mientras que la mediación es la acción docente acerca de su rol como orientador del aprendizaje y las estrategias para la promoción del aprendizaje del estudiantado. Es así que en los sistemas educativos a distancia, si bien es cierto, es fundamental el control que el estudiante ejerce sobre su proceso de aprendizaje, por cuanto debe solventar las barreras para el cumplimiento de las tareas que se le presenten; ello en plena conciencia de sus procesos de apropiación del conocimiento, así como de selección y empleo de estrategias y con motivación para aprender (Bandura, 1986; Pintrich, 1995; Pintrich \& Schunk, 2006; Zimmerman, 1989); también el rol docente en ese cometido es primordial (Vargas, 2017) para promover estrategias que posibiliten el desarrollo de habilidades para el aprendizaje autónomo, ello siempre con una mediación pedagógica adecuada (Villalobos y Vargas, 2018), la cual se define como la " la acción o actividad, intervención, recurso o material didáctico que se da en el hecho educativo para facilitar el

Estrategias docentes para la promoción del aprendizaje autónomo en estudiantes universitarios que utilizan plataformas LMS

Ana Lorena Vargas-Cubero, Gabriela Villalobos-Torres DOI: http://dx.doi.org/10.22458/caes.v10i2.2715

\section{(c) (i) (8)}


proceso de enseñanza y de aprendizaje por lo que posee carácter relacional. Su fin central es facilitar la intercomunicación entre el estudiante y los orientadores para favorecer a través de la intuición y del razonamiento, un acercamiento comprensivo de las ideas a través de los sentidos -dentro del horizonte de una educación concebida como participación, creatividad, expresividad y racionalidad-, (Fainholc, 2004, p. 3).

Por tanto, quien aprende en autonomía requiere que su proceso se cimiente en estrategias de "aprender a aprender" de manera que se dé el paso de una educación basada en la enseñanza hacia una centrada en el estudiante (Hernández y De la Cruz, 2014), pero siempre con el apoyo institucional y la orientación docente, quien se visualiza en una docencia desde el rol de facilitador y potenciador de un acceso autónomo y selectivo a la información, así como de la adquisición de un pensamiento activo, creativo y crítico, quien ejerce la docencia enfatiza en enrutar al estudiantado para que las temáticas en estudio se tornen en aprendizajes reales y con significatividad (Vargas, 2017).

Por otra parte, el aprendizaje a distancia cada vez más se viene realizado a través de lo que se conoce como LMS (Learning Management System el cual promueve el aprendizaje mediante las plataformas de aprendizaje que proveen distintas posibilidades de interacción. No obstante, el uso de estas experiencias de aprendizaje debe estar en concordancia con el rol orientador del docente, importante pero no protagónico, pues será el estudiantado quien protagonice su proceso metacognitivo, así que se puede ver el paso entre los modelos centrados en la docencia a sistemas educativos donde el conocimiento fluye desde distintas fuentes y por diversos medios, de manera tal que quien aprende no se limita a hacerlo de manera memorística, sino a aprender de forma autogestionaria con la guía del docente y a partir del uso apropiado de los recursos tecnológicos disponibles (Alcoceba, 2013).

Estrategias docentes para la promoción del aprendizaje autónomo en estudiantes universitarios que utilizan plataformas LMS

Ana Lorena Vargas-Cubero, Gabriela Villalobos-Torres DOI: http://dx.doi.org/10.22458/caes.v10i2.2715

\section{(c) (i) (8)}


En síntesis, se puede decir que probablemente el alumnado, en un modelo educativo a distancia, estudie desde su misma casa u otro lugar que le es conocido y familiar, pero el gran esfuerzo está en conocer una manera de estudiar y de aprender que quizás no ha experimentado nunca, así como en lograr relacionarse con otros (docentes, compañeras y compañeros) también de una manera diferente. Unido a ello resalta la importancia de ser autónomo en el aprendizaje y la autorregulación de ese proceso planteado para quienes estudian en entornos virtuales (Chiecher, 2014), ello como una forma de lograr el éxito académico y el logro de aprendizajes con significatividad Bixio (2005), lo anterior siempre con la guía y orientación docente.

\section{Objetivos}

\section{General:}

Analizar de qué manera las y los docentes impulsan estrategias que promueven el aprendizaje autónomo en estudiantes de un modelo educativo a distancia que utilizan Learning Management System (LMS).

\section{Específicos:}

1. Explorar el conocimiento que tienen las y los docentes implicados en el estudio acerca del aprendizaje autónomo y su promoción en estudiantes en un modelo educativo a distancia que utiliza LMS.

2. Determinar las estrategias utilizadas por docentes a distancia en asignaturas mediadas en LMS para el logro del aprendizaje autónomo en los estudiantes.

3. Determinar la pertinencia percibida por los estudiantes, acerca de las estrategias utilizadas por docentes a distancia en asignaturas mediadas en LMS para el logro del aprendizaje autónomo en los estudiantes.

4. Plantear recomendaciones para el favorecimiento del aprendizaje autónomo desde el uso de LMS en estudiantes a distancia.

Estrategias docentes para la promoción del aprendizaje autónomo en estudiantes universitarios que utilizan plataformas LMS

Ana Lorena Vargas-Cubero, Gabriela Villalobos-Torres

DOI: http://dx.doi.org/10.22458/caes.v10i2.2715

\section{(c) (i) (8)}




\section{Metodología}

El estudio es de tipo cualitativo, puesto que se buscó estudiar la realidad sin alteraciones, ni manipulaciones planeadas, por el contrario, se planteó el análisis de la situación en su contexto y tal cual como es percibido por los actores. Se enmarcó específicamente en una investigación de tipo fenomenológico que pretendió examinar las percepciones de un grupo de personas respecto de sus experiencias sobre un tema determinado (Bogdan \& Biklen, 2003), en este caso las estrategias docentes, en estudiantes a distancia de asignaturas que utilizan LMS para favorecer el aprendizaje autónomo.

Con el fin de recolectar la información, se recurrió a un cuestionario semiestructurado, validado por cuatro personas expertas en el área de la educación a distancia. El mismo fue aplicado a 38 docentes de asignaturas que utilizan LMS en estudiantes a distancia de la Escuela de Ciencias Sociales y Humanidades de la Universidad en que se realizó el estudio. Dado que es un estudio de naturaleza cualitativa las personas participantes seleccionadas no provienen de una muestra representativa, sino que fueron escogidos mediante el criterio de las investigadoras por ser personas que trabajan con la realidad estudiada.

De manera simultánea, se les planteó una única pregunta, también validada por las mismas personas expertas, a 120 estudiantes que actualmente cursan asignaturas en LMS, con el fin de indagar su opinión acerca de la mejora percibida en el desarrollo de las asignaturas a partir de las estrategias que utilizan los y las docentes para promover el aprendizaje autónomo.

Posteriormente, para asegurar que lo revelado por las personas participantes fuera beneficioso para el logro de los objetivos planteados en el estudio, se analizó la información mediante una comparación de datos provenientes de la teoría y lo declarado por los sujetos

Estrategias docentes para la promoción del aprendizaje autónomo en estudiantes universitarios que utilizan plataformas LMS

Ana Lorena Vargas-Cubero, Gabriela Villalobos-Torres

DOI: http://dx.doi.org/10.22458/caes.v10i2.2715

\section{(c) (1) (2)}


participantes. Luego de sistematizada y analizada la información, se plantearon las conclusiones pertinentes a la temática del estudio y su naturaleza cualitativa.

\section{Resultados y discusión}

Los datos se sintetizaron y ordenaron en tablas de manera sucinta, de acuerdo con lo expresado por las personas participantes, lo anterior con el fin de visualizar y comprender los temas abordados. De las tablas 1 a la 3 se hace una comparación entre la información recolectada en el campo proveniente de la consulta al profesorado, y los aspectos teóricos e institucionales; en las tablas 4 y 5 se presentan datos respecto de los resultados obtenidos a partir de la promoción de estrategias que se encaminan al aprendizaje autónomo en los entornos LMS, ello desde la percepción docente y estudiantil. Posteriormente, en la tabla 6 se compara lo manifestado por las personas participantes docentes en cuanto a la capacitación recibida en el tema de estrategias para promover el aprendizaje autónomo versus las necesidades sentidas por ellos en cuanto a la capacitación en la temática.

\section{Tablas de síntesis y sistematización de datos/ discusión}


Tabla 1.

\section{Temática en estudio}

Comprensión del significado de aprendizaje autónomo en la educación a distancia

Planteamiento teórico y/o institucional $\quad$ Información recolectada en el estudio con docentes

- Tipo de aprendizaje que se presenta cuando el estudiantado identifica problemas, establece objetivos, soluciona problemas y planifica estrategias para el logro de las metas de aprendizaje establecido (Moore, 1972).

- Se caracteriza porque "los estudiantes autónomos se evalúan y se dirigen a sí mismos...se exige que el estudiante asuma un cierto grado de dirección y control de sí mismo" (Moore, 1972, p. 87).

- La universidad plantea que este tipo de aprendizaje requiere de herramientas y estrategias que la misma institución debe proveer. Por su parte, el estudiantado es un ente activo del proceso de aprendizaje, de modo que interpreta y reinterpreta la realidad para aprender continuamente a partir de sus estructuras cognitivas (UNED, Consejo Universitario, 2004).

- En un modelo educativo a distancia, esto debe ser natural, aprender en autonomía, lo cual lleva a la generación de aprendizajes significativos y a la regulación del propio proceso de adquisición de nuevos conocimientos (UNED, Consejo Universitario, 2004).

Fuente: Elaboración propia a partir de información recolectada en el campo.

Estrategias docentes para la promoción del aprendizaje autónomo en estudiantes universitarios que utilizan plataformas LMS

Ana Lorena Vargas-Cubero, Gabriela Villalobos-Torres DOI: http://dx.doi.org/10.22458/caes.v10i2.2715

\section{(c) (1) (\$)}


En la comparación realizada, las personas participantes en el estudio, para este ítem las personas docentes, manifestaron que es un tipo de aprendizaje donde el estudiante es protagonista, gestor de su aprendizaje y con características de aprendiz autorregulado. Señalan además que la mediación docente es necesaria y pertinente para que se favorezca el proceso de aprendizaje autónomo.

Por su parte, en lo esbozado teóricamente se indica que es un aprendizaje donde quien estudia gestiona su aprendizaje (Moore, 1972), lo cual señala también a la Institución, al colocar al estudiantado como un ente activo en su proceso de cognición. Este tipo de aprendizaje es medular, pues se indica que es natural en los modelos a distancia para la generación de aprendizajes significativos (UNED, Consejo Universitario, 2004).

Existe coincidencia entre el conocimiento expresado por las personas participantes del estudio y lo que se plasma en la teoría y en documentos institucionales; no obstante, es necesario analizar más adelante si el conocimiento del concepto de aprendizaje autónomo en estudiantes a distancia, en asignaturas que se imparten en LMS, es congruente con las prácticas de docentes en los entornos para promover el tipo de aprendizaje.

Estrategias docentes para la promoción del aprendizaje autónomo en estudiantes universitarios que utilizan plataformas LMS

Ana Lorena Vargas-Cubero, Gabriela Villalobos-Torres

DOI: http://dx.doi.org/10.22458/caes.v10i2.2715

\section{(c) (i) $(5)$}


Tabla 2.

\begin{tabular}{|c|c|}
\hline \multicolumn{2}{|c|}{$\begin{array}{c}\text { Temática en estudio } \\
\text { Características que según los docentes identifican a un estudiante que aprende en } \\
\text { autonomía }\end{array}$} \\
\hline Planteamiento teórico y/o institucional & $\begin{array}{c}\text { Información recolectada en el estudio con } \\
\text { docentes }\end{array}$ \\
\hline $\begin{array}{l}\text { - Sabe aprender a aprender, quien } \\
\text { estudia adquiere cierto grado de } \\
\text { control que le permite autodirigirse y } \\
\text { autoevaluarse (Moore, 1972). } \\
\text { - Es un estudiante que participa } \\
\text { activamente y tiene control de su } \\
\text { metacognición para lograr las metas } \\
\text { educativas de modo significativo, no } \\
\text { espera pasivamente, sino que } \\
\text { gestiona su aprendizaje a partir de } \\
\text { fuentes de conocimiento dispersas y } \\
\text { diversas (UNED, Consejo } \\
\text { Universitario, 2004). }\end{array}$ & $\begin{array}{l}\text { - } \text { Disciplinado, organizado en tiempo } \\
\text { recursos y espacio } \\
\text { - } \text { Autobservación } \\
\text { - } \text { Autorregulado } \\
\text { - } \text { Independiente } \\
\text { - Seguridad en sí mismo } \\
\text { - } \text { Apmpromiso } \\
\text { - } \text { Debendizaje de manera significativa } \\
\text { - } \text { Crítico } \\
\text { - Hace consultas pertinentes. } \\
\text { - } \text { Planificado y metódico en su aprendizaje. } \\
\text { - } \text { Aprende a su propio ritmo. } \\
\text { - } \text { Prefiere en el estudio } \\
\text { - } \text { Busca aclarajo individual, no en grupos. } \\
\text { - Ordenado y responsable }\end{array}$ \\
\hline
\end{tabular}

Fuente: Elaboración propia a partir de información recolectada en el campo.

Al comparar lo expuesto por quienes ejercen la docencia con respecto a la teoría y la postura institucional, se observa como existe coincidencia en cuanto a algunas características de un estudiante que aprende en autonomía, por ejemplo, es alguien que sabe aprender y no solo es depositario de información, que tiende a la autorregulación y adquiere conocimientos significativos. No obstante, en lo señalado por los participantes se indican que es un aprendiz que prefiere trabajar solo y no en grupo, lo cual se contrapone a lo que se señala respecto de

Estrategias docentes para la promoción del aprendizaje autónomo en estudiantes universitarios que utilizan plataformas LMS

Ana Lorena Vargas-Cubero, Gabriela Villalobos-Torres

DOI: http://dx.doi.org/10.22458/caes.v10i2.2715

\section{(c) (i) (8)}


un aprendiz autónomo, que implica que no es un aprendiente aislado, sino que sabe gestionar su aprendizaje y en ello es esencial el trabajo colaborativo (Delgado \& Flores, 2009).

Además las personas participantes señalan que aprender en autonomía convierte al aprendizaje en "exponencial al haber interactividad, socialización de conocimientos y realimentación entre pares y entre estos y profesores" (ver tablas 4 y 5 ). Se puede señalar que si bien hay cierta claridad de cuáles son las características que distinguen a un aprendiz autónomo, se refleja cierta contradicción al manifestar que el estudiante que aprende en autonomía prefiere trabajar solo, cuando los estudios señalan que el trabajo colaborativo resulta positivo para el tipo de aprendiz.

Para la recolección de información esbozada en la tabla 3, además de la pregunta, se les presentó a las personas participantes las "competencias esenciales", de acuerdo con Perdomo \& Perdomo 2012, que debe poseer la persona docente a distancia en entornos virtuales para ser un verdadero apoyo pedagógico para el estudiantado. 
Tabla 3.

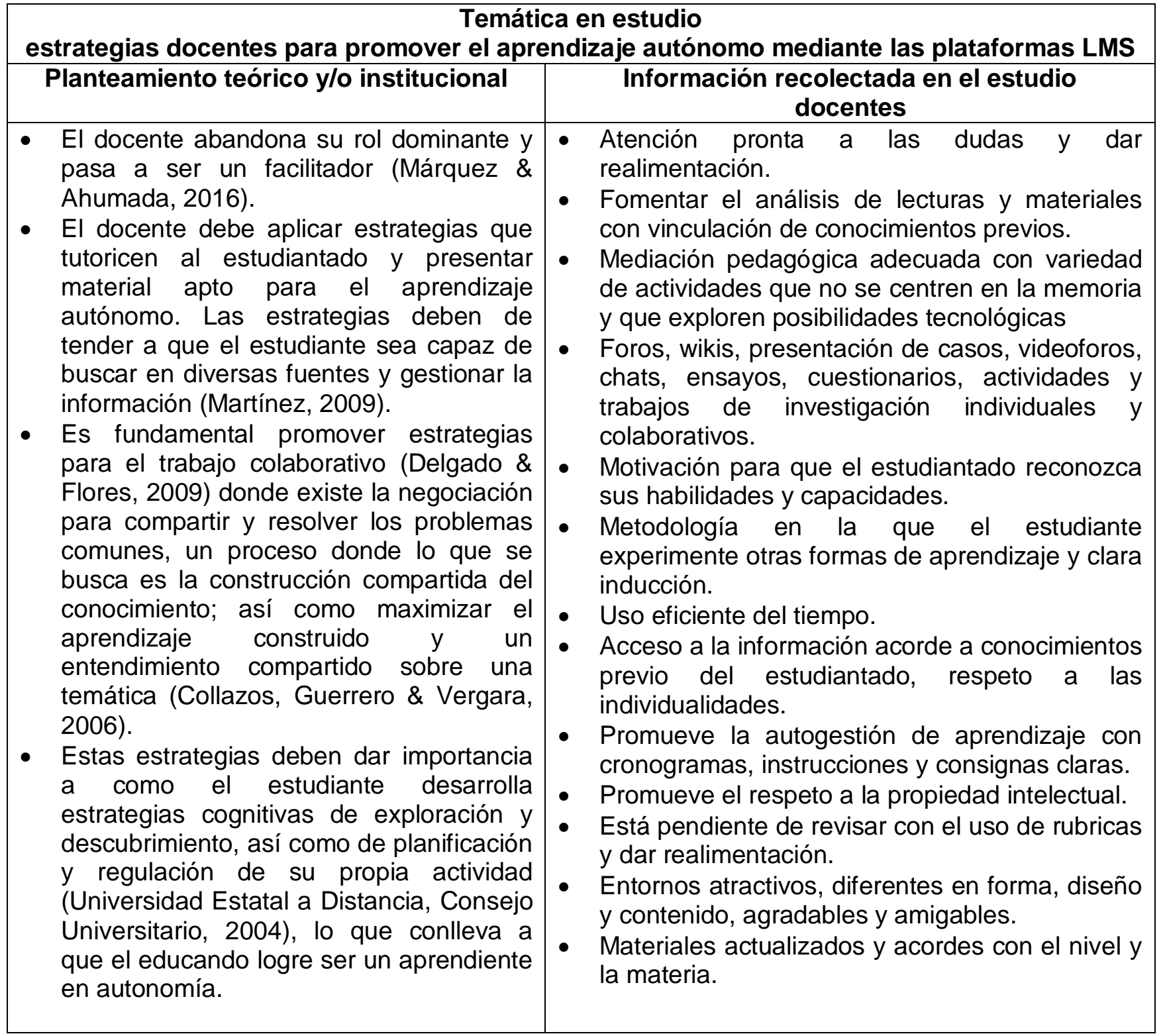

Fuente: Elaboración propia a partir de información recolectada en el campo.

Estrategias docentes para la promoción del aprendizaje autónomo en estudiantes universitarios que utilizan plataformas LMS

Ana Lorena Vargas-Cubero, Gabriela Villalobos-Torres

DOI: http://dx.doi.org/10.22458/caes.v10i2.2715

\section{(c) (i) (8)}


De acuerdo con la información de la tabla anterior, se puede apreciar como la institución y la postura teórica coinciden en señalar que las estrategias del docente a distancia para promover procesos de aprendizaje autónomo requieren, en primer lugar, cambiar el rol de un docente dominante a uno que posee características de orientador (Márquez y Ahumada, 2016, UNED, 2004). La persona docente debe entonces estimular la autogestión del aprendizaje y facilitar la autonomía (Monereo \& Pozo, 2003), por su parte los y las docentes participantes se abocaron a señalar actividades y no un modelo de docencia orientadora hacia el aprendizaje autónomo, aunque si coinciden con la institución y la teoría al planear que deben promover la autogestión del aprendizaje. 
Tabla 4.

\section{Resultados positivos o negativos producto de las estrategias utilizadas por docentes para promover en estudiantes el aprendizaje autónomo y los componentes que lo nutren en asignaturas que utilizan LMS: La perspectiva docente}

Resultados positivos

- Se puede orientar mejor al estudiante, así como darle seguimiento y tener comunicación fluida.

- Integración y puesta en práctica de nuevos conocimientos en el ejercicio profesional.

- Mayor participación y discusiones que permiten evacuar dudas y mayor aprendizaje mediante la capacidad analítica.

- Se promueve la autocorrección, autorregulación y autoaprendizaje.

- Realización de actividades con número reducido de consultas y dudas.

- Se accede a información en cualquier momento, además es más atractiva y comprensible.

- Aprendizaje exponencial al haber interactividad, socialización de conocimientos y realimentación entre pares y entre estos y profesores.

- Mayor autonomía y mejoras en el rendimiento académico.

- Se fomenta reflexión y análisis, así como mejora de habilidades de expresión escrita.

- Herramientas de evaluación variadas.

- Altos niveles de permanencia de estudiantes en las asignaturas.

- Identifican y seleccionan mejor las fuentes digitales de información.
Resultados negativos

- No utilizan ni materiales ni los foros como corresponde.

- No siempre leen instrucciones lo que hace que se reincida en los mismos errores.

- Falta de compromiso y constancia.

- Brecha digital entre los mismos estudiantes.

- Diferentes capacidades, diferentes aprendizajes.

- Tiempos inadecuados para orientarse hacia este tipo de aprendizaje.

- Subutilización de recursos facilitados por el docente.

- Plagio o fraude académico.

- Poco interés de estudiantes en la lectura profunda que implique análisis.

- Excesiva dependencia hacia el docente.

- Falta de competencias para la investigación.

- No tienen capacidad para hacerle frente a los desafíos que se le presentan.

- Acceso limitado a la plataforma por lejanía geográfica o escasez de recursos.

- Resistencia al cambio: algunos estudiantes prefieren ser evaluados de formas tradicionales.

Fuente: Elaboración propia a partir de información recolectada en el campo.

Estrategias docentes para la promoción del aprendizaje autónomo en estudiantes universitarios que utilizan plataformas LMS

Ana Lorena Vargas-Cubero, Gabriela Villalobos-Torres

DOI: http://dx.doi.org/10.22458/caes.v10i2.2715

\section{(c) (i) (8)}


Tabla 5.

\begin{tabular}{|l|l|l|}
\hline \multicolumn{4}{|c|}{ Temática en estudio } \\
Manifestaciones de estudiantes en los cursos LMS relacionados con mejora \\
percibida en el desarrollo de los mismos a partir de las estrategias implementadas \\
por el docente para promover el aprendizaje autónomo: La perspectiva del \\
estudiantado
\end{tabular}

Fuente: Elaboración propia a partir de información recolectada en el campo.

Las tablas 4 y 5 resumen la postura docente y estudiantil en cuanto a los resultados de la promoción de estrategias que busquen un aprendizaje autónomo en asignaturas que se impartan bajo parámetros LMS. Se nota como los y las docentes señalan aspectos más generales del aprendizaje de estudiantes en cuanto a que se torna más analítico, autorregulado y organizado. Los y las estudiantes por su parte se abocan a señalar ventajas que perciben en cuanto a mayor organización, resolución de dudas y atención.

Estrategias docentes para la promoción del aprendizaje autónomo en estudiantes universitarios que utilizan plataformas LMS

Ana Lorena Vargas-Cubero, Gabriela Villalobos-Torres DOI: http://dx.doi.org/10.22458/caes.v10i2.2715

\section{(c) (i) (8)}


Cabe mencionar que la pregunta planteada a estudiantes y docentes solo se generalizó en resultados sin hacer la acepción a lo negativo y lo positivo, pero estos señalaron aspectos en las dos vías, lo cual llama la atención de las investigadoras al notar como se perciben elementos negativos en un proceso de promoción de aprendizaje autónomo.

En cuanto a lo negativo los docentes señalaron lo que consideran errores $u$ omisiones del estudiantado en sus tareas y quienes aprenden manifestaron omisiones y errores docentes y estudiantiles, o tecnológicos, como los de la plataforma en sí, por lo que se ve que lo manifestado no se relacionan únicamente con el aprendizaje autónomo, sino con las acciones docentes y estudiantes.

Tabla 6.

\begin{tabular}{|c|c|c|}
\hline \multicolumn{3}{|c|}{$\begin{array}{l}\text { Temática en estudio } \\
\text { Procesos de capacitación e inducción ofrecidos por la UNED y recibidos por docentes } \\
\text { relacionados con las plataformas LMS }\end{array}$} \\
\hline Temática en estudio & $\begin{array}{c}\text { Procesos de } \\
\text { capacitación recibidos }\end{array}$ & $\begin{array}{c}\text { Recomendaciones para } \\
\text { capacitaciones }\end{array}$ \\
\hline $\begin{array}{l}\text { Procesos de capacitación e } \\
\text { inducción brindados por la } \\
\text { UNED para la aplicación de } \\
\text { estrategias como docente a } \\
\text { distancia en cursos LMS } \\
\text { para promover el } \\
\text { aprendizaje autónomo en } \\
\text { los estudiantes. }\end{array}$ & 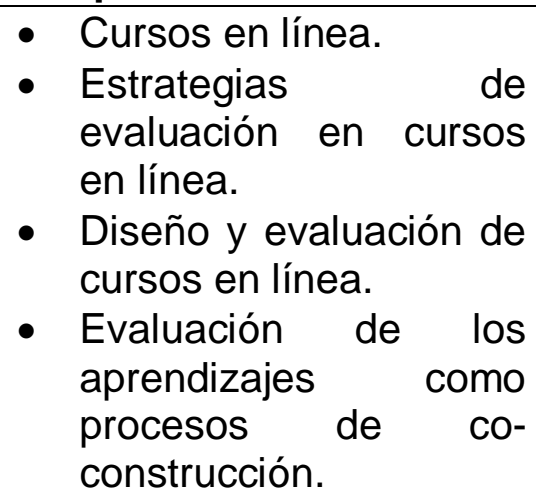 & 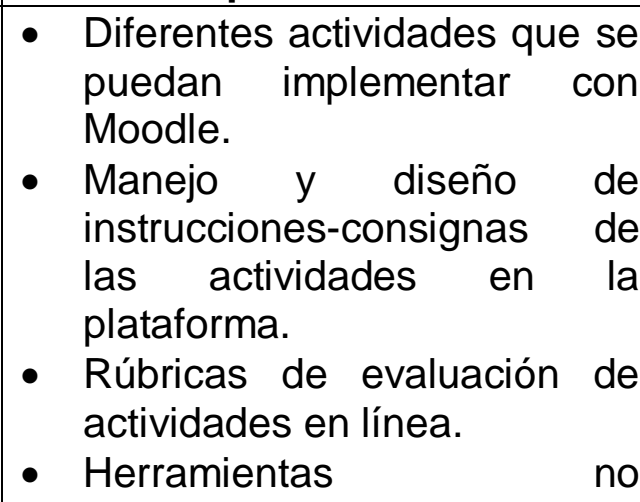 \\
\hline
\end{tabular}

Estrategias docentes para la promoción del aprendizaje autónomo en estudiantes universitarios que utilizan plataformas LMS

Ana Lorena Vargas-Cubero, Gabriela Villalobos-Torres

DOI: http://dx.doi.org/10.22458/caes.v10i2.2715

\section{(c) (1) (8)}




\begin{tabular}{|c|c|c|}
\hline $\begin{array}{l}\text { Recomendaciones de } \\
\text { capacitaciones dirigidas a } \\
\text { docentes para aplicar } \\
\text { estrategias que promuevan } \\
\text { el aprendizaje autónomo } \\
\text { en los estudiantes que } \\
\text { usan LMS. }\end{array}$ & 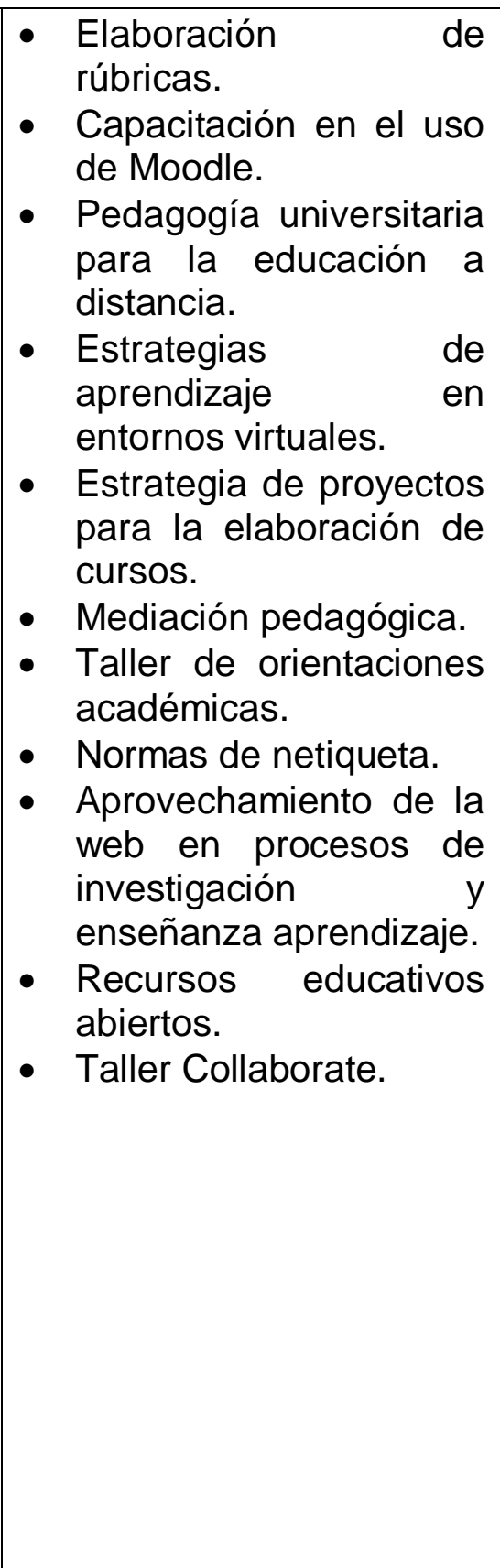 & $\begin{array}{l}\text { convencionales que ofrece la } \\
\text { plataforma. } \\
\text { - Mediación pedagógica y } \\
\text { evaluación de actividades } \\
\text { virtuales. } \\
\text { - Ajuste en el modelo de } \\
\text { asignación de tiempos, ya } \\
\text { que no es congruente el } \\
\text { asignado con la cantidad de } \\
\text { trabajo por realizar, no hay } \\
\text { tiempo para realimentar al } \\
\text { estudiante, se trabaja tipo } \\
\text { maquila. } \\
\text { - Espacios de intercambio de } \\
\text { experiencias entre docentes } \\
\text { de cursos en línea. } \\
\text { - Estrategias pedagógicas y } \\
\text { elaboración de materiales } \\
\text { para potenciar el aprendizaje } \\
\text { autónomo. } \\
\text { - Herramientas para alcanzar } \\
\text { un efectivo acompañamiento } \\
\text { en línea con los estudiantes. } \\
\text { - Teorías sobre el enfoque de } \\
\text { aprendizaje autónomo. } \\
\text { - Capacitación de aplicaciones } \\
\text { digitales para construir } \\
\text { multimedios adaptados a los } \\
\text { contenidos de cada } \\
\text { asignatura. a de aprendizaje } \\
\text { - Estrategias de abraso para poblaciones } \\
\text { autónomo } \\
\text { diversas. } \\
\text { Capacitación en estrategias } \\
\text { cognitivas. }\end{array}$ \\
\hline
\end{tabular}

Fuente: Elaboración propia a partir de información recolectada en el campo.

Estrategias docentes para la promoción del aprendizaje autónomo en estudiantes universitarios que utilizan plataformas LMS 
En la tabla 6, se presentan las capacitaciones recibidas y las recomendaciones que señalan las personas docentes como necesarias para promover estrategias que tiendan al aprendizaje autónomo. Se nota la coincidencia en temas de mediación y evaluación, lo que hace inferir que a pesar de las capacitaciones recibidas sobre las temáticas, los docentes aún perciben debilidad en esas áreas.

Las capacitaciones deben ser constantes e incluir un monitoreo o seguimiento, o al menos un espacio posterior a la finalización de las mismas para evacuar dudas o consultas específicas que surjan en la práctica y puesta en marcha de los procesos aprendidos; es decir, se requiere de un tiempo de realimentación posterior.

\section{Conclusiones}

De la investigación realizada se detallan a continuación las conclusiones.

Se percibe un claro conocimiento, por parte de los y las docentes, acerca de lo que es e implica el aprendizaje autónomo, donde se destaca al estudiante como actor principal de su proceso de aprendizaje de forma autogestionada y autorregulada, sin presencia del profesor en el proceso, más allá de la mediación pedagógica establecida en las consignas y guías dadas en el curso.

Sin embargo, el proceso como tal, en lo estricto de su definición, no se presenta en el tanto que la mediación y guía constante de la persona profesora hacia los estudiantes debe ser permanentemente, tal y como se evidencia en los resultados de las estrategias utilizadas por docentes.

La ausencia de claridad en las consignas, en las rúbricas y en la metodología de evaluación de cada asignatura ofertada a través de las LMS, se relaciona directamente con el

Estrategias docentes para la promoción del aprendizaje autónomo en estudiantes universitarios que utilizan plataformas LMS

Ana Lorena Vargas-Cubero, Gabriela Villalobos-Torres DOI: http://dx.doi.org/10.22458/caes.v10i2.2715

\section{(c) (i) (8)}


aprovechamiento o no de las mismas y el éxito académico que se obtenga. Aún existe resistencia hacia el uso de las LMS por diversas razones: temor, inseguridad o deficiencias en el uso de las TIC, o bien en la brecha tecnológica y en la plataforma en sí misma.

El aprendizaje autónomo y autorregulado es necesario para estudiantes que utilizan como método de aprendizaje el estudio a distancia y el uso de las LMS para alcanzar el mismo, pero debe de ir asociado al compromiso y la motivación de los y las estudiantes hacia su proceso de enseñanza aprendizaje, mismo que aún debe ser regulado, mediado y acompañado, ya que la comunicación para la evacuación de consultas e indicaciones claras son indispensables para que el aprendizaje autónomo sea satisfactorio. Lo anterior a pesar de tomar en consideración características de los y las estudiantes basadas en disciplina, independencia, criticidad y planificación hacia su estudio.

A su vez, se ha de destacar que el trabajo individual priva sobre el trabajo colaborativo, lo cual se contrapone a la teoría sobre el aprendiz autónomo, ya que es indispensable en una sociedad en la que se requieren habilidades para el trabajo grupal solicitadas en los mercados laborales.

Las estrategias implementadas por los y las docentes de esta investigación son variadas y responden a las diferentes necesidades que han percibido en el estudiantado. No obstante se evidencia falta de conocimiento en materia de cómo fomentar el aprendizaje autónomo en asignaturas a través de LMS; de modo que se brinden estrategias de un modelo de docencia que oriente y guíe al estudiante hacia este tipo de aprendizaje.

No se percibe que se prepare al estudiante a investigar y ahondar más en diversas fuentes acerca de las temáticas de la asignatura, así como a promover el trabajo conjunto, ya que se

Estrategias docentes para la promoción del aprendizaje autónomo en estudiantes universitarios que utilizan plataformas LMS

Ana Lorena Vargas-Cubero, Gabriela Villalobos-Torres DOI: http://dx.doi.org/10.22458/caes.v10i2.2715

\section{(c) (i) (8)}


sigue la línea de las características del estudiantado donde se prefiere el trabajo individual, tal y como se mencionó anteriormente.

La ausencia de la promoción de trabajo grupal se traduce en una minimización del potencial de conocimiento que un estudiante puede adquirir, ya que no se presenta el trabajo compartido, el análisis y el debate de diferentes ideas y conceptos teóricos que lleven a descubrimientos y replanteamiento de conocimientos.

A pesar de ello, las actividades descritas buscan que el estudiante se realimente de información, analice lecturas sugeridas y las vincule con conocimientos previos evitándose así la memorización y respuestas cerradas, así como la producción individual y el respeto a la propiedad intelectual. Para esto, la mediación pedagógica, el establecimiento de cronogramas para el uso efectivo del tiempo, las consignas y rúbricas de evaluación con información clara y pertinente son consideradas esenciales para alcanzar los logros propuestos.

Según la investigación, los resultados negativos expuestos son corregibles y dependen de todas las partes involucradas: docentes, estudiantes y la Universidad.

De parte de los estudiantes se cita que, con una adecuación efectiva en sus métodos de estudio, aprender a trabajar en grupo y minimizar resistencias, se pueden aprovechar mejor las estrategias que los y las docentes ofrecen. Por su parte, el responder con celeridad a dudas, revisar que los enlaces que se brinden estén activos, capacitarse y programar las actividades en plataforma con claridad y certeza dependiendo de la complejidad de las mismas se logra contribuir mejor desde la parte docente; finalmente, si la Universidad está

Estrategias docentes para la promoción del aprendizaje autónomo en estudiantes universitarios que utilizan plataformas LMS

Ana Lorena Vargas-Cubero, Gabriela Villalobos-Torres

DOI: http://dx.doi.org/10.22458/caes.v10i2.2715

\section{(c) (1) (8)}


vigilante de ofrecer mantenimiento a las plataformas que se utilizan, se alcanzaría una mayor autonomía en el aprendizaje.

Los procesos de capacitación son necesarios e indispensables en una temática donde el desconocimiento para fomentar el aprendizaje autónomo mediante estrategias variadas aún priva. La variedad implica desde el conocimiento en sí de la plataforma Moodle hasta aspectos de intercambio de ideas y experiencias, estrategias pedagógicas a través de LMS, aplicaciones digitales para construir multimedios, teorías sobre aprendizaje autónomo, entre otras.

A pesar de que la Universidad promueve un proceso permanente de capacitación mediante una diversa oferta de cursos, estos no son matriculados por los docentes o bien no están respondiendo a la realidad de las LMS, por lo que es pertinente ser congruente y ajustarse a las necesidades planteadas.

De no contarse con un trabajo articulado entre las partes y un mayor conocimiento de los y las docentes sobre estrategias para la promoción del aprendizaje autónomo en estudiantes universitarios que utilizan plataformas LMS, las deficiencias y carencias seguirán manteniéndose, con lo que no se contribuirá a un verdadero aprovechamiento de las TIC en los procesos de enseñanza-aprendizaje, y a la falencia de utilizar el verdadero potencial que estas poseen para fomentar la autorregulación y el aprender a aprender en los y las estudiantes.

Por todo lo anterior, se hace imperativo recomendar lo siguiente:

La pertinencia de que los y las profesoras mantengan un proceso de actualización constante, en el que se potencie el aprendizaje e implementación de estrategias que promuevan el

Estrategias docentes para la promoción del aprendizaje autónomo en estudiantes universitarios que utilizan plataformas LMS

Ana Lorena Vargas-Cubero, Gabriela Villalobos-Torres

DOI: http://dx.doi.org/10.22458/caes.v10i2.2715

\section{(c) (1) (8)}


aprendizaje autónomo de los y las estudiantes mediante las plataformas LMS, ya que es evidente la carencia que al respecto tienen.

El proceso de formación no debe de ser únicamente responsabilidad de la Universidad, sino también de cada docente que utiliza las plataformas LMS, ya que se requiere de diversidad de estrategias para satisfacer las necesidades igualmente diversas y variadas que presentan los y las estudiantes de este centro de enseñanza superior. El autoaprendizaje autónomo también debe de fomentarse entre docentes para que basados en sus propias experiencias se ofrezcan mejores servicios y se alcance un mayor éxito académico en la población estudiantil.

En una universidad a distancia el aprendizaje autónomo y autorregulado es fundamental, pero se debe de difundir e informar más al respecto desde el ingreso a esta metodología de enseñanza aprendizaje, de forma tal que el estudiante se programe y aprenda a desarrollar las destrezas y habilidades que son requeridas para alcanzar el éxito académico y un verdadero conocimiento de la materia estudiada.

Las estrategias para la promoción del aprendizaje autónomo en los estudiantes empleadas hasta el momento, a pesar de ser variadas y diversas, no están alcanzado resultados totalmente satisfactorios, aún persisten aspectos negativos que deben de corregirse y redirigirse los esfuerzos al aprovechamiento de las mismas en un medio tan versátil como el constituido por las plataformas LMS. 


\section{Referencias}

Alcoceba, H. (2013). Juventud, tecnologías de la información y cambio social. Perspectivas y escenarios para la socialización y la participación. In: CABALLERO, F. (Org.). Ciudadanía, tecnología y cultura: nodos conceptuales para pensar la nueva mediación digital. Barcelona: Gedisa.

Arguelles, D., N. Nagles (2007). Estrategias para promover procesos de aprendizaje autónomo. Colombia: Alfaomega.

Bandura, A. (1986). Social foundations of thought and action: A social-cognitive theory. Englewood Cliffs: Prentice Hall.

Betini, L. Contini, A., Castro, a. (2010). Las habilidades cognitivas en niños preescolares. Un estudio comparativo en un contexto de pobreza. Acta colombiana de psicología (13), $1,25-34$

Bixio, C. (2005). Enseñar a aprender. Construir un espacio colectivo de enseñanza aprendizaje. Argentina: Homo Sapiens Editores.

Bogdan, R. \& Biklen, S. (2003). Qualitative Research for Education: An introduction to Theories and Methods. (4⿳⺈冂䒑 edición.). New York: Pearson

Bruner, J. (1983). Charla del niño: Aprender a utilizar el lenguaje. New York: Norton.

Chiecher, A. (2014). Tres etapas en la inclusión de entornos virtuales en la enseñanza universitaria. En P. Paoloni, M. C. Rinaudo y A. González Fernández, (Comps.), Cuestiones en Psicología Educacional. Perspectivas teóricas, metodológicas y estudios de campo (325-360). Tenerife: Sociedad Latinoamericana de Comunicación Social.

Clark, R. (2001). Learning from media.Arguments, Analysis and Evidence. Greenwich, Connecticut: Information Age Publishing.

Coll, C., Mauri, T., \& Onrubia, J. (2006). Análisis y resolución de casos-problema mediante el aprendizaje colaborativo RUSC. 3 (2), 29-41. Barcelona, España.

Estrategias docentes para la promoción del aprendizaje autónomo en estudiantes universitarios que utilizan plataformas LMS

Ana Lorena Vargas-Cubero, Gabriela Villalobos-Torres

DOI: http://dx.doi.org/10.22458/caes.v10i2.2715

\section{(c) (i) (5)}


Correa, M.E., Castro, F. \& Lira, H. (2004). Estudio descriptivo de las estrategias cognitivas y metacognitivas de los alumnos y alumnas de primer año de pedagogía en enseñanza media de la universidad del bío-bío Theoria. 13, 1, 103-110. Recuperado de https://www.redalyc.org/pdf/299/29901310.pdf

Delgado, C., Flores, A., Mayoral, P. (2009). Impulso del aprendizaje autónomo por medio de actividades lúdicas en internet. Ponencia en el II Foro internacional de especialistas en enseñanza de lenguas, ciudad, país. Disponible en http://www2.ucol.mx/flex/fieel2009/fieel2009.swf

Esteban, M. \& Zapata, M. (2016). Estrategias de aprendizaje y e-Learning. Un apunte para la fundamentación del diseño educativo en los entornos virtuales de aprendizaje. Revista de Educación a Distancia. Núm. 50. Disponible en https://revistas.um.es/red/article/view/271261/198501

Fainholc, B. (2004). El concepto de mediación en la tecnología educativa apropiada y crítica. Educ.ar. Disponible en https://cmapsinternal.inmc.us/rid=1119466861556_1804172076_502/educ.ar\%20\%20 Educacion\%20y\%20TIC_\%20El\%20concepto\%20de\%20mediacion\%20en\%20la\%20t ecnologia\%20educativa\%20apropiada\%20\%20critica.pdf

Fernández, A. (2009). Las plataformas e-learning para la enseñanza y el aprendizaje universitario en Internet. Las plataformas de aprendizaje. Del mito a la realidad.Biblioteca Nueva, Madrid, 45-73.

Fundación Omar Dengo (2014). Competencias para el siglo XXI: guía práctica para promover su aprendizaje y evaluación. San José, Costa Rica: FOD.

Gallusa, J. (1998). Barreras para el aprendizaje en la educación a distancia. ERIC.

García, I. \& De la Cruz, M. (2014). Las guías didácticas: recursos necesarios para el aprendizaje autónomo. Educementro, 6 (3)

García, L. (2006). La educación a distancia: De la teoría a la práctica. Barcelona, España: Editorial Ariel Educación.

Hartman, H. y Sternberg, R. (1993). A broad BACEIS for improving thinking. Instructional Science, 21, 401-425.

Estrategias docentes para la promoción del aprendizaje autónomo en estudiantes universitarios que utilizan plataformas LMS

Ana Lorena Vargas-Cubero, Gabriela Villalobos-Torres

DOI: http://dx.doi.org/10.22458/caes.v10i2.2715

\section{(c) (i) (8)}


Lasser, W. (2018). Educación a Distancia, ¿hacia dónde te diriges?. Virtualidad, educación y Ciencia. 9 (16). Recuperado de http://revistas.unc.edu.ar/index.php/vesc

Manrique, L. (2004). El aprendizaje autónomo en la educación a distancia. Debates de Educación (Barcelona) [ponencia en línea]. Fundación Jaume Bofill, UOC.

Márquez, A. \& Ahumada, A. (eds.) (2016). La Universidad Publica en México y su compromiso social. México: ECORFAN.

Martínez, M. (2009). Análisis de las competencias desarrolladas en el aprendizaje autónomo y en el presencial: construyendo la autonomía del alumnado universitario. Revista de Enseñanza Universitaria, 34; 4-14. Disponible en http://institucional.us.es/revistas/universitaria/34/art_1.pdf

Monereo, C. \& Pozo, J. (2003). La universidad ante la nueva cultura educativa. Enseñar y aprender para la autonomía. Madrid: Síntesis.

Moore, M. (1972). La autonomía del alumno: La segunda dimensión del aprendizaje independiente. Research in Education.

Osses, S. \& Jaramillo, S. (2008). Metacognición: un camino para aprender a aprender. Estudios pedagógicos (Valdivia), 34 (1), 187-197. Disponible en https://dx.doi.org/10.4067/S0718-07052008000100011

Perdomo, Y. \& Perdomo, G. (2012). Elementos que intervienen en la enseñanza y aprendizaje en línea. Revista de innovación educativa, (4), 1, 66-75. Disponible en http://www.udgvirtual.udg.mx/apertura/index.php/apertura/issue/view/48

Pintrich, P. (1995). Entendiendo el aprendizaje autorregulado. New directions for Teaching and Learning, 63, 3-12

Pintrich, P. \& Schunk, D. (2006). Motivación en contextos educativos. Teoría, investigación y aplicaciones. Madrid, España: Pearson

Salas \& Umaña (2011). Análisis del debate sobre el efecto de los medios tecnológicos en los procesos de aprendizaje. Revista Actualidades Investigativas en Educación. (11) 1, 122.

Disponible

en https://www.researchgate.net/publication/280964019_Analisis_del_debate_sobre_el_e fecto_de_los_medios_tecnologicos_en_los_procesos_de_aprendizaje

Estrategias docentes para la promoción del aprendizaje autónomo en estudiantes universitarios que utilizan plataformas LMS

Ana Lorena Vargas-Cubero, Gabriela Villalobos-Torres DOI: http://dx.doi.org/10.22458/caes.v10i2.2715

\section{(c) (i) $(5)$}


Simonson, M., Smaldino, S., Albright, M. \& Zvacek, S. (2006). Teaching and learning at a distance: Foundations of distance education ( $3^{\text {ra }}$ ed.) Upper Saddle River, NJ: Prentice Hall.

Universidad Estatal a Distancia, Consejo Universitario (2004). Modelo Pedagógico. San José: EUNED.

Valenzuela, J. (2000). Los tres autos del Aprendizaje: Aprendizaje estratégico en educación a distancia. Revista de la Escuela de Graduados en Educación 2 (1). Recuperado de file://C:/Users/avargas/Documents/Los\%20tres\%20autos\%20del\%20aprendizaje.\%20 Aprendizaje\%20estrategico\%20en\%20educacion\%20a\%20distancia.pdf

Valle, A., González, R. \& Núñez, J.C. (1996). Dimensiones cognitivo-motivacionales y aprendizaje autorregulado. Revista de Psicología, 14(1). Recuperado de http://ezproxybib.pucp.edu.pe/index.php/psicologia/article/view/4559/4538importancia

Varela-Ruiz, MUNAM. (2009). Aprendizaje independiente y aprendizaje colaborativo en educación médica. 72, 4, 222 - 227. México: UNAM

Vargas, A.L. (2017). El Papel del Docente en el Aprendizaje Autorregulado de Estudiantes Universitarios a Distancia. Disertación Doctoral. USA: Nova Southeastern University

Vargas, A.L. \& Villalobos, G. (2018). El uso de plataformas virtuales y su impacto en el proceso de aprendizaje en las asignaturas de las carreras de Criminología y Ciencias Policiales, de la Universidad Estatal a Distancia de Costa Rica. Educare 22 (1) 20-39. Recuperado de http://dx.doi.org/10.15359/ree.22-1.2

Writt, K. \& Perkins, D. (2008). Aprender a aprender. Recuperado de http://www.macalester.edu/geology/wirth/CourseMaterials.html

Zimmerman, B. J. (1989). Una visión sociocognitiva del aprendizaje académico autorregulado. Journal of Educational Psychology, 81(3), 329-339.

Zimmerman, B. \& Martinez-Pons, M. (1986). Desarrollo de una entrevista estructurada para evaluar en los estudiantes el uso de estrategias de autorregulación del aprendizaje. American Educational Research Journal, 23, 614-628. Recuperado de: http://aer.sagepub.com/cgi/content/abstract/23/4/614 